\title{
Energy Efficiency Joint Cell Selection and Power Allocation for HetNets
}

\author{
HAMIT abdoulaye issak \\ Chongqing Key Labs of Mobile \\ Communications Technology, \\ Chongqing University of Posts and \\ Telecommunications \\ Chongqing, China \\ teguenabd@163.com
}

\begin{abstract}
Aiming at the heterogeneity of transmission performance in heterogeneous networks (HetNets) consisting of multiple cells, an optimal cell selection and power allocation scheme is proposed to improve QoS and QoE of users. For one UE case, the proposed scheme selects the optimal cell corresponding to the maximum energy efficiency of the UE. In the case of multiple UEs, the QoS and power constrained energy optimization problem is equivalently transformed into two sub-problems, which are power allocation of each UE-cell pair and cell selection of UEs. The two sub-problems are solved respectively by applying Lagrange dual method and Kuhn-Munkres (K-M) algorithm. Numerical results demonstrate the efficiency of the proposed algorithm.
\end{abstract}

\section{Keywords}

HetNets; Cellular network; Energy efficiency; Lagrange; KuhnMunkre

\section{INTRODUCTION}

The cost of energy is expected to increase in the future. With the growing concerns on this issue, we need to take a critical look at saving energy by reducing consumption. Energy efficiency has therefore attracted a lot of attention from both academia as well as the cooperative world. Henceforth, telecom operators and academia are trying to consider network energy efficiency as a key performance indicator in today's cellular networks. Telecom companies increase capacity in order to meet the explosive demand in data traffic as well as maintain needed Quality of Service (QoS), try to have commercially deployed small cells technology. Hence, traditional homogeneous Macro Base Stations (MBSs) in Cellular networks are changing into heterogeneous Network (HetNet). This approach brings to the table a significant improvement in the entire network capacity and achievable of user data rate and also offers a promising way to reduce the amount of energy consumed in the entire network.

Even though HetNet goes a long way to reduce energy consumption of the entire network [1-3], it is worth noting that, the number of BSs required is highly related with the energy consumption of the network. On the one hand, some related works focus on transmit power optimization based cell selection in

Permission to make digital or hard copies of all or part of this work for personal or classroom use is granted without fee provided that copies are not made or distributed for profit or commercial advantage and that copies bear this notice and the full citation on the first page. To copy otherwise, to republish, to post on servers or to redistribute to lists, requires prior specific permission and/or a fee.

Mobimedia 2017, July 13-14, Chongqing, People's Republic of China

Copyright (๑) 2017 EAI 978-1-63190-156-0
- For single UE case, a two-step algorithm is proposed, which firstly conducts optimal power allocation sub algorithm on the cell and then chooses the optimal cell corresponding to the maximum energy efficiency.

- Extending to the multiple UEs, the optimization problem is divided into two sub-problems., namely power allocation sub-problem and cell selection one of UEs, and Lagrange dual method and $\mathrm{KM}$ algorithm are respectively utilized to solve the above two sub-problems.

\section{PROPOSED SCHEME}

Consider the scenario that consists of a mixture of disparate mobile access technologies and protocols such as micro cells and low-power nodes, for example, Micro, Pico, Femto as shown in Figure 1. Relay node and remote radio head are added to macro cells whose transmission power ranges from $5 \mathrm{~W}$ and $40 \mathrm{~W}$ [9-11] to enhance capacity and coverage. In today's telecom world, HetNet has become an attractive means of expanding mobile network capacity.

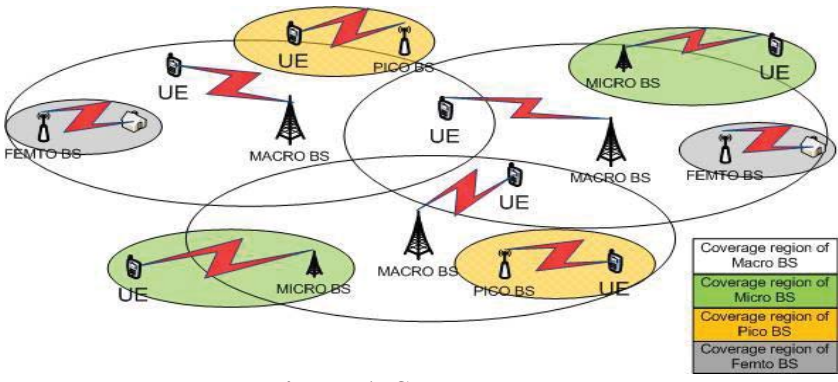

Figure 1. System model

In the model, we assume that orthogonal spectrum sharing scheme is applied for the cells, i.e., various spectrum is allocated to different cells, hence no inter-cell interference exists. Furthermore, in order to avoid intra- cell interference, different time-frequency resource blocks are allocated to UEs of each cell. The channel is flat-fading channel with the gain being a constant at transmitting time interval and the noise is additive white Gaussian noise.

\section{JOINT OPTIMIZATION OF CELL SELECTION AND POWER ALLOCATION}

This paper mainly focuses on the energy efficiency of UE and proposes an energy efficient optimization scheme which selects the optimal cell corresponding to the maximum energy efficiency of the UE subject to QoS and power constraints of the UEs. Therefore, the formulated optimization problem is equivalently transformed into two sub-problems, namely the power allocation sub-problem of each UE-cell pair and cell selection sub-problem of UEs, respectively. Consequently, the two sub-problems are 
solved respectively by applying Lagrange dual method and KuhnMunkres (K-M) algorithm.

\subsection{Candidate Cell Selection Scheme}

Based on the system model, the data rate between UE and cell can be expressed as [12]:

$$
R_{i j}=B_{j} \log \left(1+\frac{P_{i j}^{T} \cdot h_{i j}}{\sigma^{2}}\right)
$$

where $R_{i j}$ denotes the achievable data rate of the $i$-th UE when accessing the $j$-th cell, $B_{j}$ is the bandwidth of the $j$-th cell. $P_{i j}^{T}$ indicates the transmit power of the $i$-th UE when accessing the $j$ th cell, $h_{i j}$ and $\sigma^{2}$ respectively denote the channel gain and the noise power of the link from the $i$-th UE to the $j$-th cell. Therefore, the data rate constraint is defined as:

$$
R_{i j} \geq R_{i}^{\min }, 1 \leq \mathrm{j} \leq N
$$

where $R_{i}^{\min }$ is the minimum data rate requirement of the $i$-th UE.

Transmission power constraint can be derived as:

$$
P_{i j}^{T} \leq P_{i}^{\max }, 1 \leq i \leq M, 1 \leq j \leq N
$$

where $P_{i j}^{T}$ denotes the allowable transmit power of the $i$-th UE.

Combine with the equations above, we can achieve:

$R_{i j}^{\max }=B_{j} \log \left(1+\frac{P_{i j}^{T} \cdot h_{i j}}{\sigma^{2}}\right) \geq R_{i}^{\min }, 1 \leq i \leq M, 1 \leq j \leq N$

Accordingly, we denote $\Phi$ as the set of all cells and $\Phi_{i}$ refers to the set of candidate cells of the $i$-th UE. Hence, $\Phi_{i}$ can be denoted as:

$$
\Phi_{i}=\left\{C_{j} \mid R_{i j}^{\max } \geq R_{i}^{\min }, C_{j} \in \Phi\right\}
$$

\subsection{Proposed Joint Optimization Scheme: Single UE Case}

In this case, we focus on the joint cell selection and power allocation for the UE with consideration of energy efficiency of UE, which is defined as:

$$
\eta_{j}=\frac{R_{1, i}}{P_{1, j}^{T}+P_{c}}
$$

where $\eta_{j}$ denotes the energy efficiency of the $i$-th UE when accessing the $j$-th cell, and $P_{c}$ is the circuit consumption power of the UE, which is assumed to be a constant for all the UEs in the work.

To stress this problem, we propose a two-step algorithm which consists of both power allocation and cell selection sub algorithms. In detail, we first conduct optimal power allocation sub algorithm on the $j$-th cell, i.e., optimizing $\eta_{j}$ in terms of
$P_{i j}^{T}$ to obtain the maximum $\eta_{j}$, which is denoted by $\eta_{j}^{*}, C_{j} \in \Phi_{i}, 1 \leq i \leq M$.

Afterwards, apply optimal cell selection sub algorithm, i.e., choosing the optimal cell corresponding to the maxium $\eta_{j}^{*}$. Furthermore, solute the optimal allocation problem of the $j$-th cell $C_{j} \in \Phi_{i}, 1 \leq i \leq M$, which is modeled as:

$$
\begin{aligned}
& \max \eta_{j} \\
& \text { s.t. }\left\{\begin{array}{l}
R_{1, j} \geq R_{1}^{\min } \\
P_{1, j}^{T} \geq P_{1}^{\max }
\end{array}\right.
\end{aligned}
$$

Given $\eta_{j}^{*}, C_{j} \in \Phi_{i}, 1 \leq i \leq M$, we can then conduct optimal cell selection sub algorithm through which the $j^{*}$ th cell offering the maximum $\eta_{j}^{*}$ is selected as the optimal cell among all $C_{i} \in \Phi_{i}, 1 \leq j \leq N$, so

$$
C_{j}=\arg _{C_{i} \in \Phi_{i}} \max \left(\eta_{j}^{*}\right)
$$

where $j^{*}$ th is the optimal $j$-th cell.

\subsection{Proposed Joint Optimization Scheme: Multiple UEs Case}

We jointly consider the performance of all UEs, and propose an optimal cell selection and power allocation scheme that attains the performances optimization of all UEs.

In the case of multiple UEs, the joint Energy Efficiency of UEs is given by:

$$
\eta=\sum_{i=1}^{M} \sum_{j=1}^{N} \beta_{i j} \eta_{i j}
$$

where $\eta_{i j}$ denotes the corresponding energy efficiency when the $i$-th UE access to the BS in the $j$-th cell. $\beta_{i j} \in\{0,1\}$ denotes the selection binary variable of the $i$-th UEs and $j$-th cell respectively. Thus, $\eta_{i j}$ can be expressed as:

$$
\eta_{i j}=\frac{R_{i j}}{P_{i j}^{T}+P_{c}}
$$

where $R_{i j}$ indicates the data rate requirement of the $i$-th UE assigned to the $\mathrm{BS}$ in the $j$-th cell. The constraint on selection variable $\beta_{i j}$ can be expressed as: 


$$
\begin{aligned}
& \sum_{j=1}^{N} \beta_{i j} \leq 1,1 \leq i \leq M \\
& \sum_{i=1}^{M} \beta_{i j} \leq 1,1 \leq j \leq N
\end{aligned}
$$

Combined with the above data rate as well as transmission power constraints, the optimization model is defined as:

$$
\begin{gathered}
\max _{\beta_{i j} P_{i j}^{T}} \sum_{i=1}^{M} \sum_{j=i}^{N} \beta_{i j} \eta_{i j} \\
\text { s.t. }\left\{\begin{array}{c}
\beta_{i j} \in\{0,1\}, 1 \leq i \leq M, 1 \leq j \leq N \\
P_{i j} \geq 0,1 \leq i \leq M, 1 \leq j \leq N \\
\sum_{j=1}^{N} \beta_{i j} \leq 1,1 \leq i \leq M \\
\sum_{i=1}^{N} \beta_{i j} \leq 1,1 \leq j \leq N \\
\beta_{i j}=0, \text { if } C_{j} \in \Phi_{i}, 1 \leq i \leq M, 1 \leq j \leq N \\
R_{i} \geq R_{i}^{\min }, 1 \leq j \leq N \\
\sum_{j=1}^{N} \beta_{i j} P_{i j}^{T} \leq P_{i}^{\max }, 1 \leq i \leq M
\end{array}\right.
\end{gathered}
$$

\subsubsection{Sub-Problem 1: Iterative Algorithm-based} Optimal Power Allocation Scheme

The optimal power allocation of UE can be conducted through solving the following optimization problem.

$$
\begin{aligned}
& \max _{P_{i j}^{T}} \eta_{i j} \\
& \text { s.t. }\left\{\begin{array}{c}
R_{j} \geq R_{j}^{\min }, 1 \leq i \leq M, 1 \leq j \leq N \\
P_{i j}^{T} \geq 0,1 \leq i \leq M, 1 \leq j \leq N \\
P_{i j}^{T} \leq P_{i}^{\max }, 1 \leq i \leq M, 1 \leq j \leq N
\end{array}\right.
\end{aligned}
$$

Obviously, the initial problem is converted into a linear binary fractional problem, which can be conveniently solved by the iterative algorithm [13].In order to solve the problem, we introduce $q$ as the efficiency of the considered system:

$$
q=\frac{R_{i j}}{P_{i j}^{T}+P_{c}}
$$

We denote $q^{*}$ as the optimal value of $q$, that is the maximum energy efficiency, so

$$
q^{*}=\frac{R_{i j}^{*}}{P_{i j}^{T^{*}}+P_{c}}=\max _{P_{i j}^{T}} \frac{R_{i j}}{P_{i j}^{T}+P_{c}}
$$

It can be proved that the maximum energy efficiency $q^{*}$ is achieved if and only if the following condition is satisfied:

$$
R_{i j}\left(P_{i j}^{T}\right)-q^{*}\left(P_{i j}^{T}+P_{c}\right)=0
$$

The optimization problem formulated in (15) can be converted into the following one:

$$
\begin{aligned}
& \max _{P_{i j}^{T}} R_{i j}\left(p_{i j}^{T}\right)-q\left(P_{i j}^{T}+P_{c}\right) \\
& \text { s.t. }\left\{\begin{array}{c}
R_{j} \geq R_{j}^{\min }, 1 \leq i \leq M, 1 \leq j \leq N \\
P_{i j} \geq 0,1 \leq i \leq M, 1 \leq j \leq N \\
P_{i j}^{T} \leq P_{i}^{\max }, 1 \leq i \leq M, 1 \leq j \leq N
\end{array}\right.
\end{aligned}
$$

For a given energy efficiency $q$, the problem is transformed into a convex problem of power allocation, which can be solved via Lagrange method. Based on the mentioned Lagrange method, the energy efficiency $q$ can be updated, and the process of solving locally optimal energy efficiency and transmit power alternatively can be conducted by an iterative algorithm, as shown in Table I.

\section{TABLE I. Iterative Resource Allocation Algorithm}

1. Initialization: the maximum number of iterations $U_{\max }$ and the maximum tolerance $\rho_{1}$.

2. Set the energy efficiency $q=0$ and iteration index $u=0$

3. repeat Main Loop

4. For a give $q$, solve for $P_{i j}^{T^{\prime}}$

5. If $R_{i j}^{\prime}-q^{\prime}\left(P_{i j}^{T^{\prime}}+P_{c}\right) \leq \rho_{1}$

6. Convergence $=$ true

7. return $\left\{P_{i j}^{T^{*}}\right\}=\left\{P_{i j}^{T^{\prime}}\right\}$

8. else

9. set $q^{*}=\frac{R_{i j}}{P_{i j}^{T}+P_{c}}$ and $u=u+1$

10. Convergence $=$ false

11. end if

12. until convergence $=$ true or $u=U_{\max }$

Algorithm 1 illustrates that the iterative operation must be undertaken to get the $q^{*}$ until the algorithm converges. Considering separate iteration the optimization power allocation problem is solved for a given $q$.

\subsubsection{K-M Algorithm Based Cell Selection Scheme}

Given $P_{i j}^{T^{*}}$, the optimal cell selection problem can be solved. For convenience, we define:

$$
\eta_{i j}^{*}=\frac{R_{i j}\left(P_{i j}^{T^{*}}\right)}{P_{i j}^{T^{*}}+P_{c}}
$$

Accordingly, the optimal cell selection problem can be further transformed into: 


$$
\begin{aligned}
& \max _{\beta_{i j}} \sum_{i=1}^{M} \sum_{j=i}^{N} \beta_{i j} \eta_{i j}^{*} \\
& \beta_{i j} \in\{0,1\}, 1 \leq i \leq M, 1 \leq j \leq N \\
& \sum_{i=1}^{M} \beta_{i j} \leq 1,1 \leq i \leq M \\
& \sum_{j=1}^{N} \beta_{i j}, 1 \leq j \leq N
\end{aligned}
$$

Typically the optimization problem formulated in (19) is a linear binary matching problem. Viewing cell selection constraints of UEs, this optimization problem can be expressed as an optimal matching problem in bipartite graph and can then be solved by an algorithm such as Kuhn Munkres (K-M).

The application of K-M algorithm is preceded which has already been explained in the previous section. In the application of K-M algorithm, a weighted complete bipartite graph $G$ with a partition $G=\{X, Y ; E\}$ is formulated in an attempt to achieve optimal cell selection, where $X$ denotes the set of UEs, i.e., $X=\left\{U E_{1}, U E_{2}, \cdots, U E_{M}\right\}$, while $\mathrm{Y}$ denotes the set of cells, that is, $Y=\left\{C_{1}, C_{2}, \cdots, C_{N}\right\}$. In the weighted complete bipartite graph, the definition of the edge weight $E\left(U E_{i}, C_{j}\right)$ is expressed as follows:

$$
w\left(U E_{i}, C_{j}\right)=\eta_{i j}^{*}
$$

The procedure of solving the optimal cell selection subproblem based on K-M algorithm can be described as follows:

(1) Start with an arbitrary feasible vertex label $l$, determine $G_{l}$ and choose an arbitrary matching $K$ in $G_{l}$.

(2) If $K$ is the maximum matching for $G$, then $K$ is optimal and the optimization problem is solved. Otherwise, the label $p$ having not being allotted by the distribution $K$ is selected in $G_{l}$. Set $S=\{X\}$ and $T=\Phi$.

(3) Let $N_{G_{l}}(S)$ denotes the points set connecting with $S$ in $G_{l}$. If $N_{G_{l}}(S) \neq T$, go to step (2), otherwise, $N_{G_{l}}(S)=T$.

(4) Construct a new label $l^{\prime}$ by:

$$
l^{\prime}=\left\{\begin{array}{l}
l(x)-\Delta, x \in S \\
l(x)+\Delta, x \in T \\
l(x), \text { otherwise }
\end{array}\right.
$$

The above process continues until an equal complete match sub-graph is obtained.

\section{NUMERICAL RESULTS}

Assuming the BS and UE are located in a square region with the length of $100 \mathrm{~m}$, and their numbers are 5 . The summary of other simulation parameters are provided in Table II. We averaged the simulation results over 1000 independent adaptation processes. Different position actualization of the UEs in each adaptation process is performed.

Table II. System Parameters

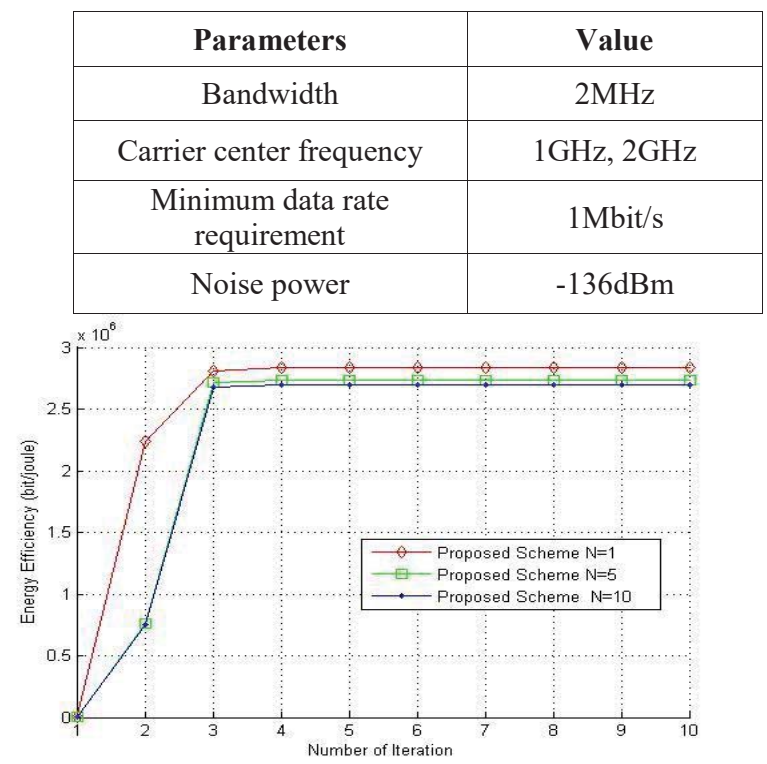

Figure 2. Energy Efficiency versus the number of Iteration

Figure 2 shows the energy efficiency of UEs versus the number of iterations obtained from the proposed algorithm. For comparison purposes, we examine the results for different numbers of the UEs whose maximum transmit power $P^{\max }$ is chosen as $0.1 \mathrm{~W}$. From the figure, we can see that the energy efficiency converges within a small number of iterations for the three cases. Comparing the results obtained from different numbers of UEs, we can see that the energy efficiency of the UEs decreases a little bit with the increase of the number of UEs. This is due to resource competition among UEs.

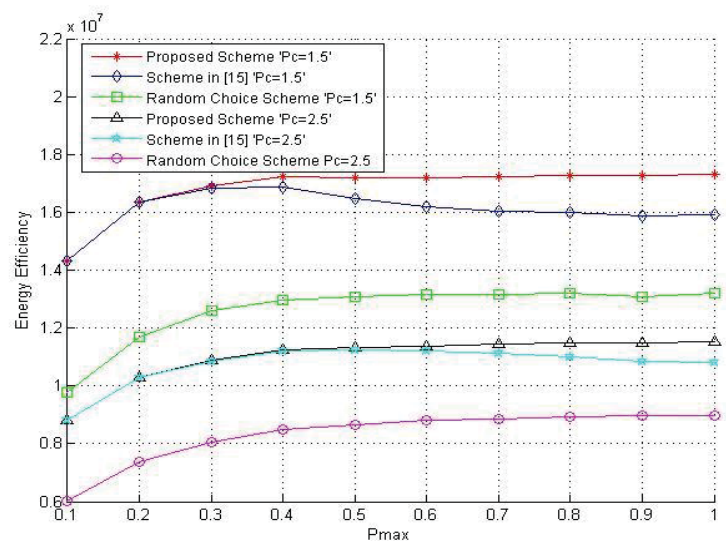

Figure 3. Energy Efficiency versus Maximum Transmit Power

Figure 3 shows the energy efficiency versus the maximum transmission power of UEs for different circuit power consumption. It can be seen that the energy efficiency decreases with the increase of the circuit power consumption. Meanwhile, in the case of small $P_{\max }$, the energy efficiency increases with $P_{\max }$ for all the schemes, indicating a larger power threshold is 
desired for achieving the maximum energy efficiency. However, as $P_{\max }$ reaches to a certain value, the energy efficiency of the proposed scheme converges to a fixed value for the transmit power less than $P_{\max }$ has resulted in the optimal energy efficiency, which will no longer vary with $P_{\max }$. On the other hand, Since the scheme proposed in [15] aims to achieve the maximum system utility, corresponding to the maximum data rate and the maximum transmit power in turn, we can see from the figure that after achieving the maximum energy efficiency, the energy efficiency decreases with the increase of $P_{\max }$, which is resulted from the large power consumption of the UEs. Comparing the results obtained from proposed scheme, the scheme proposed in [15] and random choice scheme, we can see that the proposed scheme outperforms the scheme proposed in [15] and the random choice scheme.

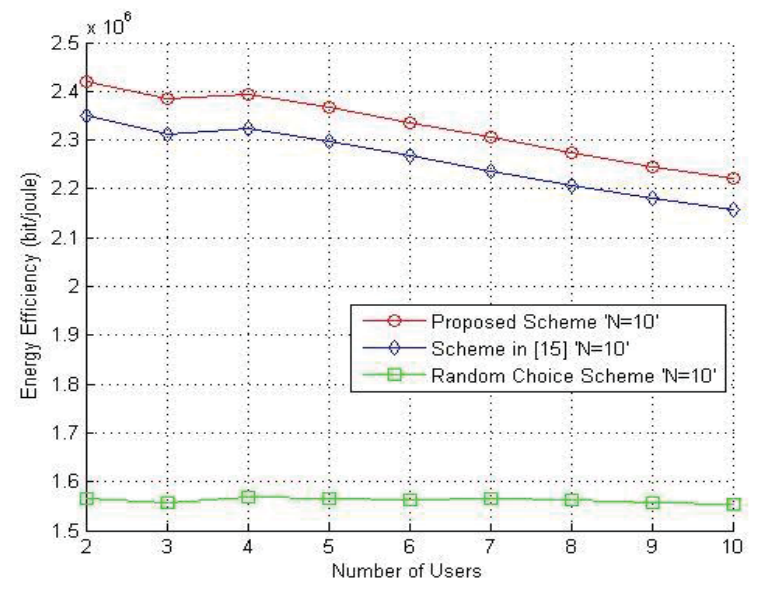

Figure 4. Energy Efficiency versus number of UEs

In figure 4 we plot energy efficiency versus the number of UEs. It is clear that the energy efficiency decreases with the increase of UEs due to resource competition among UEs. To examine the performance of the proposed scheme, we plot the results of the proposed scheme based on K-M algorithm, and those of the proposed scheme in [15] based on utility maximization in [15] and random choice algorithm. It can be seen that the proposed scheme offers a better performance compared with both the proposed scheme in [15] and random choice algorithm.

\section{CONLUSION}

An energy-efficiency joint cell selection and power allocation scheme is proposed for HetNets. In order to reduce the computational complexities, we introduce candidate cell selection in the case of one UE, and extend it to multiple UEs. Then we formulate the total energy efficiency of UEs and design an optimization problem which maximizes the total energy efficiency subject to QoS and power constraints of the UEs. The formulated optimization problem is equivalently transformed into two sub-problems, Finally, the two sub-problems are solved by Lagrange dual method and Kuhn-Munkres (K-M) algorithm respectively. Our numerical results demonstrate the efficiency of our proposed algorithm.

\section{REFERENCES}

[1] Zhang X, Su ZW, Yan Z, Wang WB (2013) Energy efficiency study for two-tier heterogeneous networks (hetnet) under coverage performance constraints. Mobile Networks and Applications, 18(4): 567-577.

[2] Ye QY, Rong BY, Chen YD (2012) Towards an optimal user association in heterogeneous cellular networks. IEEE Global Communications Conference, 4143-4147.

[3] Benmimoune A, Khasawneh FA, Kadoch, M (2015) User Association for HetNet Small Cell Networks. IEEE International Conference on Future Internet of Things and Cloud, 113-117.

[4] Smolyar L, Bergel I, Messer H (2009) Unified approach to joint power allocation and base assignment in nonorthogonal networks. IEEE Vehicular Technology Transactions, 58(8):4576-4586.

[5] Silva A, Tembine H, Altman E, Debbah M (2013) Optimum and equilibrium in assignment problems with congestion: mobile terminals association to base stations. IEEE Transactions on Automatic Control, 58(8): 2018-2031.

[6] Nguyen DHN, Le-Ngoc T (2013) Joint beamforming design and base-station assignment in a coordinated multicell system. Iet Communications, 7(10):942-949.

[7] Yu G, Jiang Y, Xu L, Li GY (2015) Multi-objective energy-efficient resource allocation for multi-rat heterogeneous networks. IEEE Journal on Selected Areas in Communications, 33(10):2118-2127.

[8] Jiang J, Peng M, Zhang K, Li L (2013) Energy-efficient resource allocation in heterogeneous network with crosstier interference constraint. IEEE International Symposium on Personal, Indoor and Mobile Radio Communications, 168-172.

[9] Lin ZH (2012) Building heterogeneous networks of green base stations on TI's KeyStone II architecture. White Paper.

[10] Chai R, Li J, Guo Z, Zhang H (2014) Poster: An energyefficient subchannel and power allocation scheme for OFDMA cellular heterogeneous networks. International Conference on Communications and NETWORKING in China, 644-645.

[11] Vanghi TV, Naga AK, Ji B (2010) LTE-Advanced: Heterogeneous Networks. IEEE Wireless Conference.

[12] Ng DWK, Lo ES, Schober R (2012) Energy-efficient resource allocation in ofdma systems with large numbers of base station antennas. IEEE Transactions on Wireless Communications, 11(9):3292-3304.

[13] Dinkelbach W (1967) On nonlinear fractional programming. Management Science, 13(7):492-498.

[14] Damnjanovic A, Montojo J, Wei Y, Ji T(2011). A survey on 3gpp heterogeneous networks. IEEE Wireless Communications, 18(3):10-21.

[15] Shen K, Yu W (2014) Distributed pricing-based user association for downlink heterogeneous cellular networks. IEEE Journal on Selected Areas in Communications, 32(6):1100-1 